\title{
Patterns of Daily Motor-Symptom Control with Carbidopa/Levodopa Enteral Suspension Versus Oral Carbidopa/Levodopa Therapy in Advanced Parkinson's Disease: Clinical Trial Post Hoc Analyses
}

\author{
Rajesh Pahwa (D) - Jason Aldred (D) - Niodita Gupta • Emi Terasawa • Viviana Garcia-Horton • \\ David R. Steffen · Prasanna L. Kandukuri • Vivek S. Chaudhari · Yash J. Jalundhwala • \\ Yanjun Bao · Pavnit Kukreja · Stuart H. Isaacson (D)
}

Received: December 15, 2021 / Accepted: January 26, 2022 / Published online: February 22, 2022

(C) The Author(s) 2022

\begin{abstract}
Introduction: A clinical trial in advanced Parkinson's disease (APD) has established the superiority of carbidopa/levodopa enteral suspension (CLES) in reducing total patient "off" time (OFF) and increasing total "on" time without troublesome dyskinesia (ON-woTD) over orally administered immediate-release carbidopa/levodopa tablets (IR-CL). However, temporal patterns of these improvements throughout the waking day have not been examined. In this analysis, time to ON-woTD after waking and patterns of motor-symptom
\end{abstract}

Supplementary Information The online version contains supplementary material available at https:// doi.org/10.1007/s40120-022-00332-0.

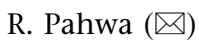

University of Kansas Medical Center, 3901 Rainbow Boulevard, Kansas City, KS 66160, USA

e-mail: rpahwa@kumc.edu

J. Aldred

Selkirk Neurology, Spokane, WA, USA

N. Gupta - P. L. Kandukuri · V. S. Chaudhari . Y. J. Jalundhwala · Y. Bao · P. Kukreja AbbVie Inc., North Chicago, IL, USA

E. Terasawa · V. Garcia-Horton · D. R. Steffen Analysis Group, Inc., New York, NY, USA

S. H. Isaacson

Parkinson's Disease and Movement Disorders

Center, Boca Raton, FL, USA control throughout the waking day were compared between CLES and IR-CL.

Methods: Post hoc analyses of APD patient-diary data from the phase 3 randomized controlled trial were used to compare changes in time to ON-woTD after waking, motor-symptom control throughout the waking day, occurrence of extreme fluctuations between OFF and "on" with troublesome dyskinesia, and motor-state transitions with CLES versus IR-CL from baseline to week 12 .

Results: The sample included 33 CLES-treated and 30 IR-CL-treated patients. Among the CLES group, the percentage of patient days achieving ON-woTD within $30 \mathrm{~min}$ of waking was three times higher at week 12 versus baseline (33\% vs. $11 \%, p=0.0043)$; no significant change occurred with IR-CL. When the waking day was divided into four 4-h periods, CLES versus IR-CL treatment produced significantly greater reductions in OFF during three periods, and two periods had increased ON-woTD. Fewer CLEStreated patients had extreme fluctuations at week 12 (3\% vs. $23 \%, p=0.0224)$ compared to IR-CL-treated patients. From baseline to week 12, CLES-treated patients had greater reductions in the average number of motorstate transitions compared to IR-CL-treated patients $(-1.6, p=0.0295)$.

Conclusion: CLES-treated patients experienced a more rapid onset of ON-woTD after waking and greater consistency of ON-woTD 
throughout their waking day than IR-CL-treated patients.

\section{PLAIN LANGUAGE SUMMARY}

In advanced Parkinson's disease, patients' motor-symptom states (such as "on" time without troublesome dyskinesia [good "on" time] and "off" time), and the timing at which they occur, can impact patients' quality of life and ability to complete activities of daily living. Carbidopa/levodopa enteral suspension is administered continuously into the jejunum, potentially reducing some of the motor-state variation that is common with orally administered carbidopa/levodopa, including delayed "on" time after waking and transitions between "off" and "on" throughout the day. In post hoc analyses of clinical trial data, patterns of motorstates across the waking day were compared between carbidopa/levodopa enteral suspension and orally administered immediate-release carbidopa/levodopa at week 12. Outcomes included time to good "on" after waking; occurrence of extreme fluctuations between "off" time and "on" time with troublesome dyskinesia; time in each motor-state during 4-h intervals across the day; and frequency of motor-state transitions. Three times as many carbidopa/levodopa enteral suspension-treated patients achieved good "on" within 30 min of waking after 12 weeks versus baseline, whereas no significant change was observed for the orally administered immediate-release carbidopa/levodopa group. Compared to orally administered immediaterelease carbidopa/levodopa-treated patients, fewer carbidopa/levodopa enteral suspensiontreated patients experienced extreme fluctuations, had greater reductions in motor-state transitions, and greater reductions in duration of "off" during three of the four intervals in the day. These findings provide a first look at the impact of carbidopa/levodopa enteral suspension on motor-state patterns throughout the day, and suggest that carbidopa/levodopa enteral suspension provides more consistent motor-symptom control and predictable benefit throughout the day than orally administered carbidopa/levodopa.

Keywords: Carbidopa/levodopa; Carbidopa/ levodopa enteral suspension; Carbidopa/ levodopa intestinal gel; Duopa; Duodopa; Dyskinesia; Parkinson's disease

\section{Key Summary Points}

\section{Why carry out this study?}

Carbidopa/levodopa enteral suspension (CLES) has demonstrated superiority to immediate-release carbidopa/levodopa tablets (IR-CL) in reducing OFF time and increasing "on" time without troublesome dyskinesia (ON-woTD) in patients with advanced Parkinson's disease, but the temporal patterns and consistency of these improvements throughout a waking day have not been examined.

This post hoc analysis compared patterns of motor-symptom control during the day, time to ON-woTD after waking, and occurrence of extreme motor-state fluctuations between CLES and IR-CL at week 12 of a randomized controlled trial.

\section{What was learned from the study?}

CLES-treated patients experienced a more rapid onset of ON-woTD after waking, greater consistency of motor-symptom control, and fewer patients had extreme motor-state fluctuations throughout their waking day than IR-CL-treated patients.

These findings suggest that CLES provides more consistent symptom control and more predictable benefit throughout the day for patients with advanced Parkinson's disease than IR-CL. 


\section{DIGITAL FEATURES}

This article is published with digital features, including a video, to facilitate visualization of motor state transitions across the day at baseline and week 12 by treatment group. To view digital features for this article go to https://doi. org/10.6084/m9.figshare.19070354.

\section{INTRODUCTION}

Medication management of advanced Parkinson's disease (APD) involves a balance between reducing "off" time (OFF) and dyskinesia [1]. With oral carbidopa/levodopa (CL) therapies and advancing disease, patients continue to experience OFF time, "on" time with troublesome dyskinesia (ON-wTD), dose failures, earlymorning OFF, and delayed time to ON. These often arise from a combination of factors including gastrointestinal dysmotility, the short half-life of orally administered immediate-release (IR) levodopa, and progressive striatal denervation with loss of buffering capacity and pulsatile stimulation of postsynaptic receptors [2-4]. This can result in disruptive troublesome dyskinesia that can emerge when levodopa levels are higher (peak-dose dyskinesia) $[5,6]$. Troublesome dyskinesia can also emerge at the beginning and end of a levodopa dose with lower levodopa levels (diphasic dyskinesias). Types of OFF include end-of-dose wearing OFF [7], early-morning akinesia [8], delayed ON, and dose failures. OFF episodes occurring with oral levodopa therapy can significantly impact activities of daily living (ADL) and quality of life (QoL), including patients physical, social, and mental well-being [9-12].

Unlike orally administered CL, carbidopa/ levodopa enteral suspension (CLES) is administered via a PEG-J tube that is connected to a portable pump. The pump delivers CLES into the jejunum, where levodopa transport primarily occurs, bypassing gastric dysmotility and avoiding issues with delayed gastric emptying common in APD [13-16]. Through continuous infusion directly to the jejunum, CLES enables steadier blood plasma levels with less variable absorption of levodopa compared to orally administered levodopa, potentially providing more continuous dopaminergic stimulation.

CLES has demonstrated greater efficacy over orally administered immediate-release carbidopa/levodopa tablets (IR-CL) in reducing OFF and increasing ON without troublesome dyskinesia (ON-woTD, "Good ON") [17, 18]. A randomized, double-blinded, double-dummy, parallel group clinical trial assessed CLES versus IR-CL over 12 weeks of treatment [17]. Overall, CLES provided a larger decrease in mean total OFF during the 16-h waking day than IR-CL (change from baseline of $-4.04 \mathrm{~h}$ for CLES vs. $-2.14 \mathrm{~h}$ for IR-CL; OFF difference between CLES and IR-CL, $-1.91 \mathrm{~h}[95 \% \mathrm{CI}-3.05$ to $-0.76] ; p=0.0015)$, and a larger increase in mean total ON-woTD than IR-CL (change from baseline of $+4.11 \mathrm{~h}$ for CLES vs. $+2.24 \mathrm{~h}$ for IR-CL; ON-woTD difference between CLES and IR-CL, $+1.86 \mathrm{~h} \quad[95 \% \quad \mathrm{CI} \quad 0.56-3.17]$; $p=0.0059$ ). However, the temporal pattern of improvements in motor-symptom control throughout the day has not been examined previously. For the present analyses, we hypothesize that CLES treatment compared to IR-CL provides a more predictable motorsymptom response through reductions of earlymorning akinesia, quicker onset to initial ONwoTD, and fewer transitions between OFF and ON states.

\section{METHODS}

\section{Study Design and Participants}

Data were used from CLES's pivotal trial (ClinicalTrials.gov identifiers NCT00660387 and NCT0357994) that was conducted at 26 centers in Germany, New Zealand, and the USA between January 2009 and October 2011. Trials conformed with the Helsinki Declaration of 1964 , as revised in 2013, and were approved by an independent ethics committee or institutional review board at each participating study site. All patients provided informed consent prior to study participation. Patients were adults (aged 30 years or older) with APD complicated by OFF periods under optimized medical therapy. Patients completed a Parkinson's disease 
(PD) home diary, which captured five possible motor states-OFF, ON without dyskinesia, ON with non-troublesome dyskinesia, ON-wTD, and asleep-experienced by 30-min increments over 3 days before each study visit. The second and third days prior to each visit were used in the analyses in order to ensure wake-up time was calculable for all patient diary days. Details about eligibility criteria and study design were previously reported $[2,17]$. The present analysis included all patients with evaluable 24-h PD home-diary assessment of motor status in the trial. For these patients, outcomes were compared between the CLES and IR-CL groups at baseline and week 12 .

\section{Outcome Measures}

The patient diary data were used to construct the outcome measures for this analysis. As with previous studies, the motor states ON without dyskinesia and $\mathrm{ON}$ with non-troublesome dyskinesia were merged and renamed as $\mathrm{ON}$ without troublesome dyskinesia (ON-woTD) for the present analyses [3]. For each patient diary day, the waking day was defined as a 16 -h period starting at wake-up time, corresponding to the daily time period of the continuous infusion of CLES [17]. Wake-up time was defined for each diary day as the start of the patient's first $2 \mathrm{~h}$ reported with no sleep after $3 \mathrm{AM}$ [18].

Four outcomes were studied. The first of these was time to reach ON-woTD after waking, to help evaluate morning akinesia [9]. This outcome was evaluated via the percentage of patient days achieving ON-woTD at four separately assessed time points-the first four halfhours of the day, namely, the time points 30, 60,90 , and 120 min after waking, respectively. The second outcome was consistency of motorsymptom control throughout the day. This was evaluated using two separately assessed measures-(1) average duration in OFF and (2) average duration of ON-woTD-during the 16-h waking day, as split into four equal intervals of $4 \mathrm{~h}$ each: from waking to $4 \mathrm{~h}$ after waking, from 4 to $8 \mathrm{~h}$ after waking, from 8 to $12 \mathrm{~h}$ after waking, and from 12 to $16 \mathrm{~h}$ after waking. The third outcome was presence of extreme fluctuations, which was measured as the percentage of patients with occurrence of an extreme fluctuation (i.e., a transition from OFF to ON-wTD) during any of their waking days. The fourth outcome was stability of motor states throughout the day and was assessed in two ways. The first definition measured the average number of motor-state transitions across the patient's waking days. The second definition assessed the proportion of patients with (1) 3 or fewer transitions, (2) 3-6 transitions, (3) 6-9 transitions, (4) 9-12 transitions, and (5) more than 12 transitions on average across their waking days.

\section{Statistical Analyses}

For time to onset of ON-woTD after waking, comparisons were conducted between the treatment groups at each time point using $z$ tests; $z$ tests also assessed change from baseline (CFB) to week 12 within the treatment groups. For consistency of motor-symptom control and number of motor-state transitions during the waking day, Wilcoxon rank-sum tests compared the treatment groups at each time point; Wilcoxon signed-rank tests assessed CFB to week 12 within each treatment group. Treatment effects (i.e., CFB) were compared between the treatment groups using analysis of covariance (ANCOVA) models including effects for treatment, country, and with the corresponding baseline value as a covariate. For extreme fluctuations, chi-squared tests compared the treatment groups at each time point, and McNemar's tests assessed CFB to week 12 within each treatment group. For the distribution of motor-state transitions during the waking day, Fisher's exact tests compared the treatment groups at each time point, and multinomial logit models assessed CFB to week 12 within each treatment group with clustering for repeated observations.

\section{Sensitivity Analyses}

Sensitivity analyses assessed and compared average use of CL (evaluated as daily plus rescue CL use) between the two treatment groups at week 12 for the following three time periods: (1) 
first $4 \mathrm{~h}$ after awakening, (2) during the patient's 16-h waking day, and (3) nighttime (defined as from pump-off time to at least $2 \mathrm{~h}$ prior to pump-start time for the next day). Comparisons were performed using Wilcoxon rank-sum tests. Average CL use during the first $4 \mathrm{~h}$ after waking and during the patient's waking day were assessed to understand whether improvements throughout the day could be attributed to differences in dosage between the treatment groups. Average CL use during the nighttime was assessed to understand whether differences in nighttime CL use could explain outcome differences between CLES and IR-CL, particularly for time to onset of ON-woTD after waking.

\section{RESULTS}

\section{Study Participants and Baseline Characteristics}

Of 69 patients in the full analysis sample, six patients were excluded from this analysis. Three patients (one from the CLES group and two from the IR-CL group) were excluded because of missing data at week 12, and three patients (two from the CLES group and one from the IR-CL group) were excluded because of one or more invalid diary data on days that would be used in the calculation of all outcomes. The final analysis sample included 63 patients, 33 CLES-treated patients and 30 IR-CL-treated patients.

Baseline characteristics of the evaluable patients are summarized in the Supplementary Material Table S1. There were no significant differences in demographic, clinical, or treatment characteristics between the two treatment groups at baseline. At baseline, CLES- versus IR-CL-treated patients were, on average, 64.0 versus 64.9 years of age, and male patients comprised $66.7 \%$ versus $70.0 \%$ of these groups, respectively. CLES-treated patients had an average PD duration of 10.2 years versus 11.8 years in the IR-CL group. The numeric difference in baseline daily dose of levodopa between the two groups was small and nonsignificant.

\section{Time to Onset of ON-woTD After Waking}

CLES was associated with greater improvements in onset of ON-woTD after waking than IR-CL. Among the CLES group, $11 \%$ of patient days achieved ON-woTD within 30 min of waking at baseline, whereas at week $12,33 \%$ achieved ONwoTD within $30 \mathrm{~min}$ of waking ( $p=0.0043)$. In the IR-CL group, there was no significant change from baseline in the percentage of patient days achieving ON-woTD within $30 \mathrm{~min}$ of waking (Fig. 1). Comparing the two treatment groups, after 12 weeks, $49 \%$ of the patient days among the CLES group achieved ON-woTD within $1 \mathrm{~h}$ of waking. However, only $27 \%$ of the patient days among the IR-CL group achieved ON-woTD within $1 \mathrm{~h}$ after waking, and it took $2 \mathrm{~h}$ for $49 \%$ of the patient days from the IR-CL group to achieve ON-woTD (Fig. 1).

\section{Consistency of Motor-Symptom Control: Duration of OFF Throughout the Day}

CLES treatment was associated with significant reductions in OFF from baseline to week 12 that were observed for all four periods throughout the 16-h waking day. By contrast, IR-CL treatment produced significant decreases in OFF from baseline to week 12 for only two of the four 4 -h periods (Fig. 2a). The reductions from baseline to week 12 in the average amount of OFF experienced during each 4-h period for CLES-treated patients ranged from 34 to $52 \mathrm{~min}$ across the four periods, whereas these reductions ranged from 4 to $46 \mathrm{~min}$ across the four periods for IR-CL-treated patients (Fig. 2a). The greater range in IR-CL demonstrated less consistent effect throughout the day.

Comparative analyses controlling for country and baseline values showed that CLES produced significantly greater reductions in OFF from baseline to week 12 than IR-CL for the first $12 \mathrm{~h}$ after waking. The greatest relative reduction in OFF was in the first $4 \mathrm{~h}$ after wakingduring this period, the reduction in OFF among CLES patients was 82 min greater than the OFF reduction among IR-CL-treated patients $(p=0.0008) \quad$ (Supplementary Material Table S2a). 


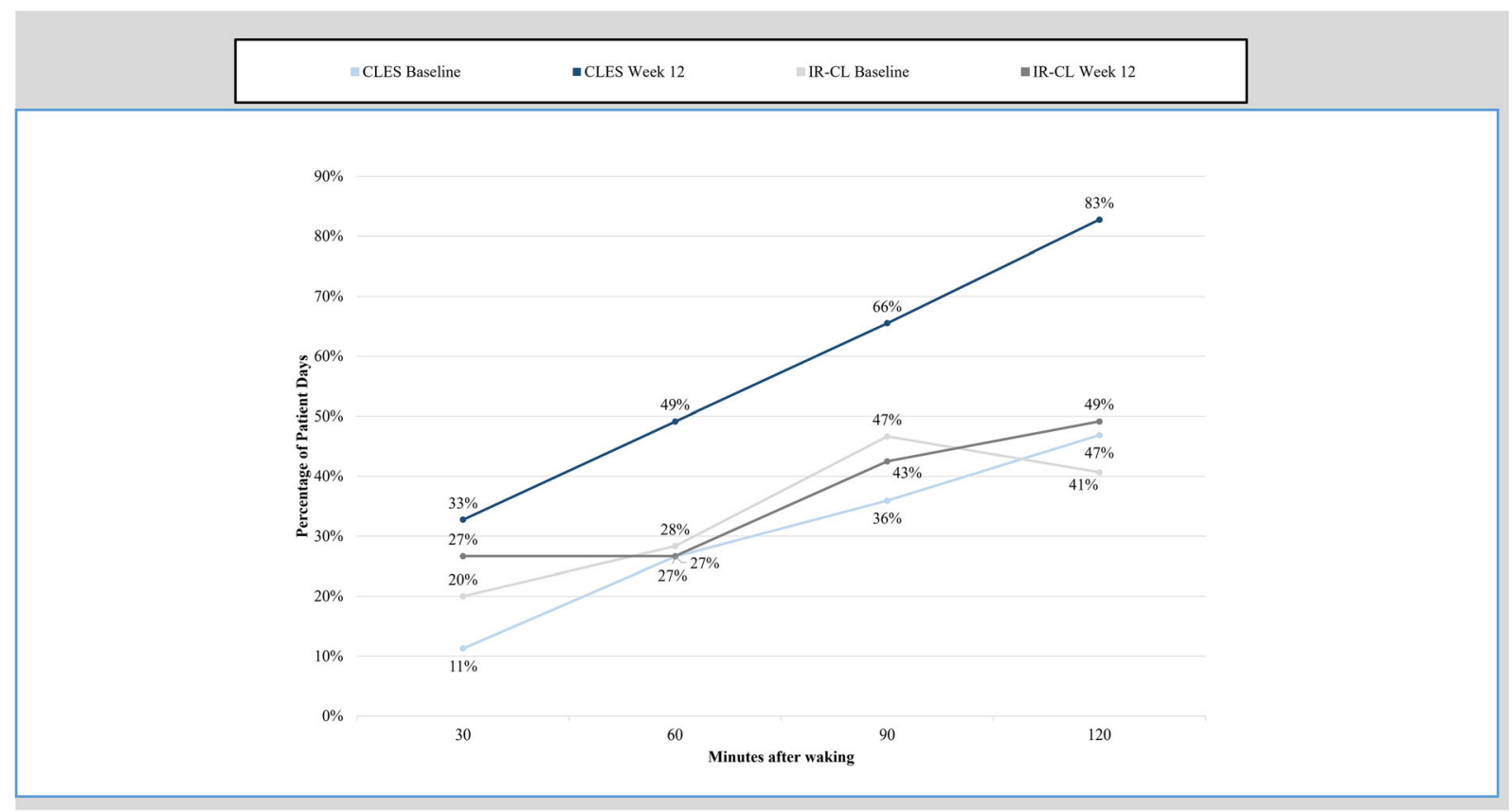

Fig. 1 Percentage of patient days achieving "on” time without troublesome dyskinesia (ON-woTD) after waking

At week 12, the percentage of patient days with OFF among the CLES group was numerically lower across almost all of the 30-min intervals throughout the day compared to that of the IR-CL group (Fig. 2b). Additionally, for nearly half of the 30-min intervals throughout the day (i.e., 15 of 32 intervals), the odds of experiencing OFF versus another motor state were more than twice as high for the IR-CL group than for the CLES group. Figure 3, which shows individual patient days for the analysis sample, further reinforces the CLES group's lower OFF across all periods of the waking day at week 12 relative to the IR-CL group.

\section{Consistency of Motor-Symptom Control: Duration of ON-woTD Throughout the Day}

Similar to OFF, significant improvements in ON-woTD from baseline to week 12 were observed across all four periods of the day in CLES-treated patients, but for only two of the four periods of the day in IR-CL-treated patients. In the CLES-treated patients, increases in the average duration of ON-woTD from baseline to week 12 ranged from 33 to $58 \mathrm{~min}$ across the four 4 -h periods, while in the IR-CLtreated patients these ranged from 8 to $46 \mathrm{~min}$ across these periods, suggesting less consistent improvement in ON-woTD throughout the day (Fig. 2c). Figure S1 shows a dynamic visualization of the increase in ON-woTD time and reduction in OFF time across the waking day for CLES-treated patients at week 12 by depicting the motor states that patients experienced during each half-hour of the day.

Comparative analyses controlling for country and baseline values showed that CLES produced significantly greater increases in ONwoTD than IR-CL for two of the four periods of the day. Compared to IR-CL, CLES-treated patients had a 44-min greater increase in $\mathrm{ON}$ woTD during the first $4 \mathrm{~h}$ after waking $(p=0.0058)$, and a 35 -min greater increase in ON-woTD during the 4 -h period from the 8 to $12 \mathrm{~h}$ post-waking $(p=0.0240)$ (Supplementary Material Table S2a).

At week 12, the percentage of patient days with ON-woTD among the CLES group was 


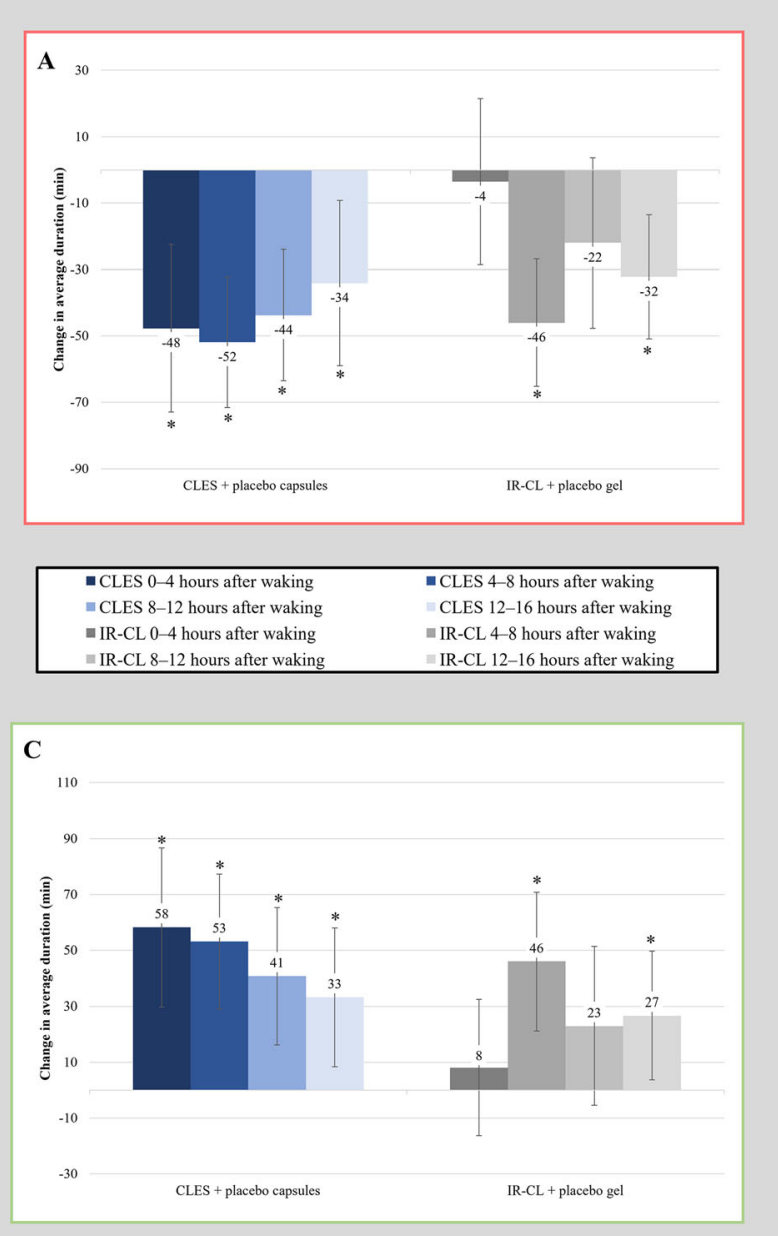

Fig. 2 a Change in "off" time (OFF) from baseline to week 12 by time of the day. $\mathbf{b}$ Percentage of patient days with "off" time (OFF) at week 12. c Change in "on" time without troublesome dyskinesia (ON-woTD) from

numerically greater across almost all of the 30-min intervals throughout the day compared to that of the IR-CL group (Fig. 2d). Additionally, for more than a third of the 30-min intervals throughout the day (i.e., 12 of 32 intervals), the odds of experiencing ON-woTD versus another motor-state was more than twice as high for the CLES group than for the IR-CL group. Patient motor states across the day may also be seen in Video 1 in the online/HTML version of the manuscript, or by following the digital features link under the abstract.
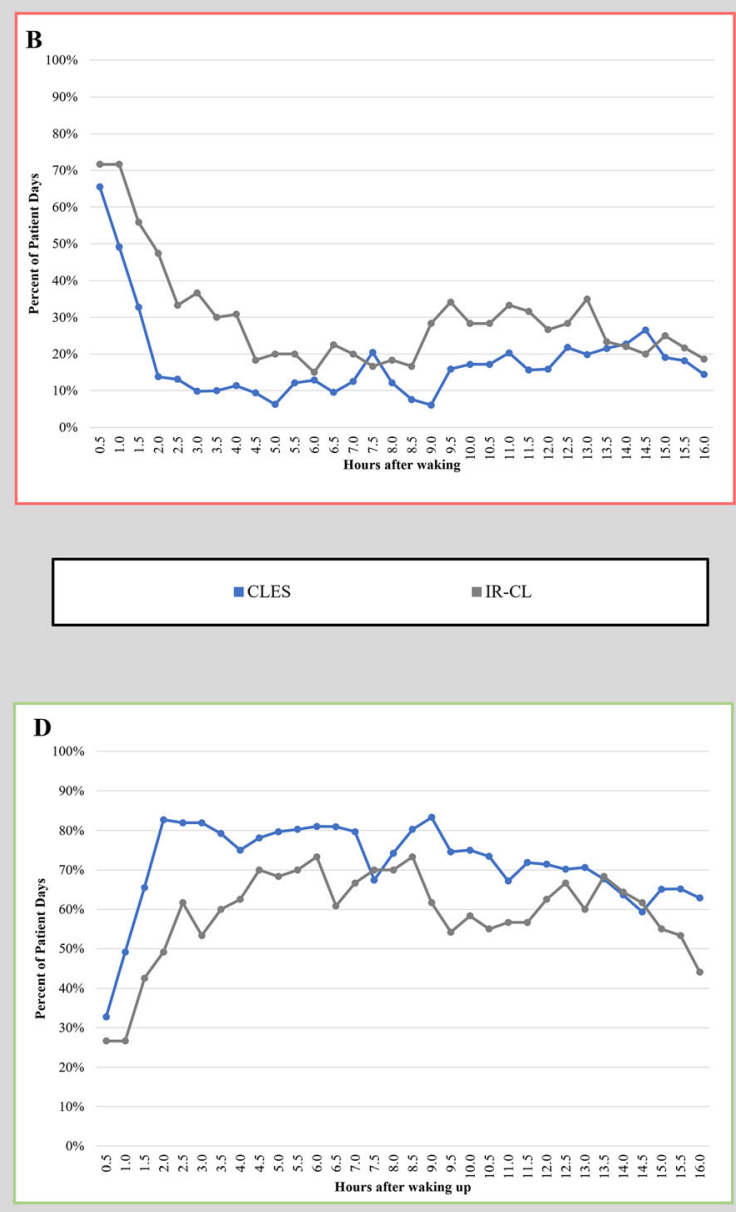

baseline to week 12 by time of the day. $\mathbf{d}$ Percentage of patient days having "on" time without troublesome dyskinesia (ON-woTD) at week 12

\section{Extreme Fluctuations}

At baseline, there was no significant difference between the treatment groups in the proportion of patients experiencing extreme fluctuations at baseline $(21.2 \%$ in the CLES group vs. $36.7 \%$ in the IR-CL group, $p=0.1751$ ). At week 12 , only one CLES-treated patient experienced an extreme fluctuation compared to seven IR-CLtreated patients $(3.0 \%$ vs. $23.3 \%$, respectively, $p=0.0224)$. The change from baseline to week 12 in the percentage of patients experiencing an extreme fluctuation was significant 


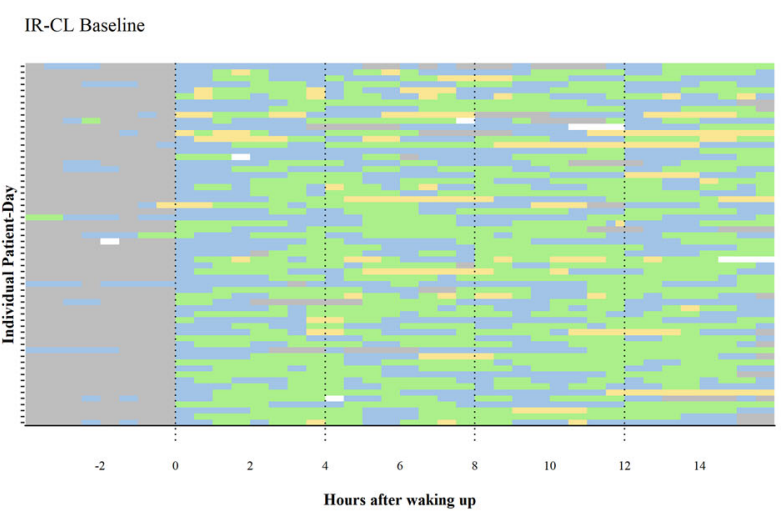

CLES Baseline

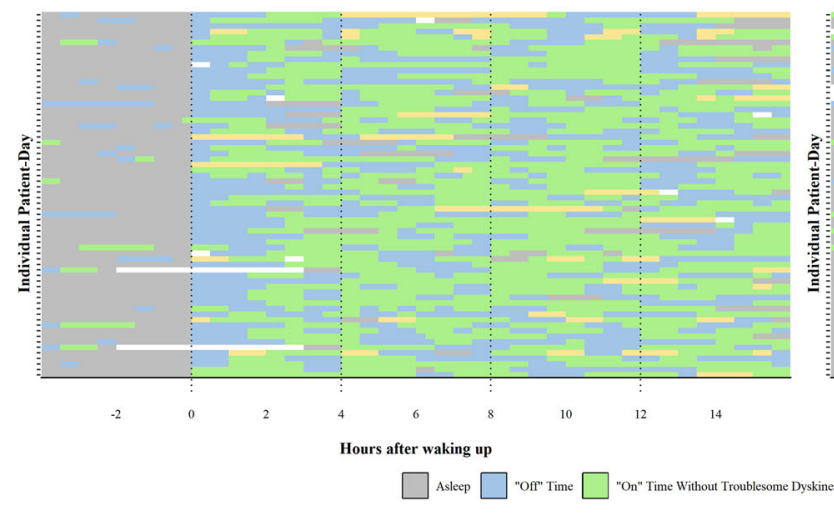

Fig. 3 Individual patient diary plots

with CLES $(-18.2 \%, p=0.0143)$, but not IR-CL $(-13.3 \%, p=0.2059)$ (Supplementary Material Table S2b).

\section{Motor-State Transitions During the Waking Day}

At baseline, both treatment groups experienced approximately eight motor-state transitions on average during their waking day, and there was no significant difference in the distribution of motor-state transitions between the groups. At week 12 , the percentage of patients with three or fewer transitions on average during their waking day was three times greater in the CLES group than in the IR-CL group (39.4\% vs. $13.3 \%$, respectively). Moreover, at week 12 , $87.9 \%$ of CLES-treated patients had six or fewer transitions on average during their waking day compared to only $56.6 \%$ of IR-CL-treated patients (Fig. 4).

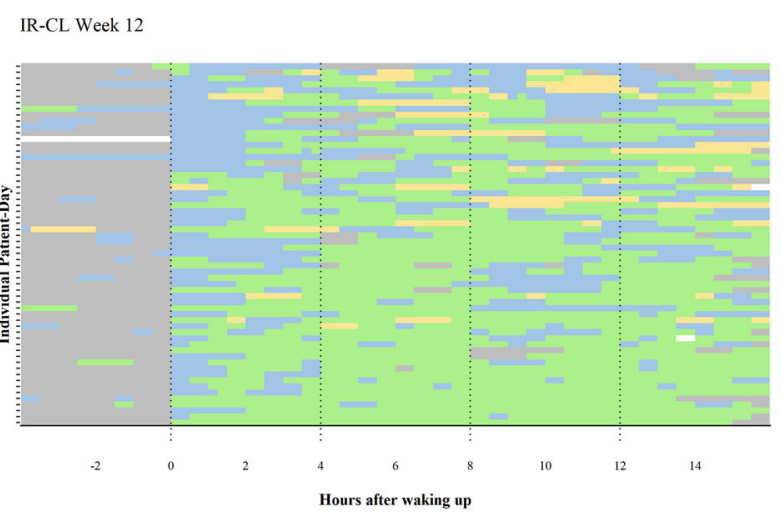

CLES Week 12

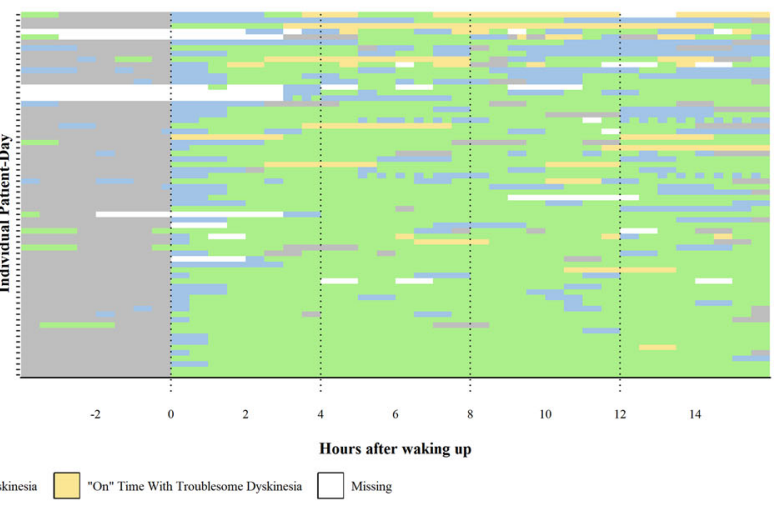

Comparative analyses showed that from baseline to week 12 , the reduction in the average number of motor-state transitions during the waking day was greater in CLES-treated patients than in IR-CL-treated patients $(-3.5$ versus -2.0 , respectively). This treatment difference was significant (LS mean difference, $-1.6 ; p=0.0295)$.

\section{Sensitivity Analyses}

There were no significant differences in morning, daytime, or nighttime CL between the CLES and IR-CL groups at week 12. Results for these analyses can be found in the Supplementary Material Table S3. 
A

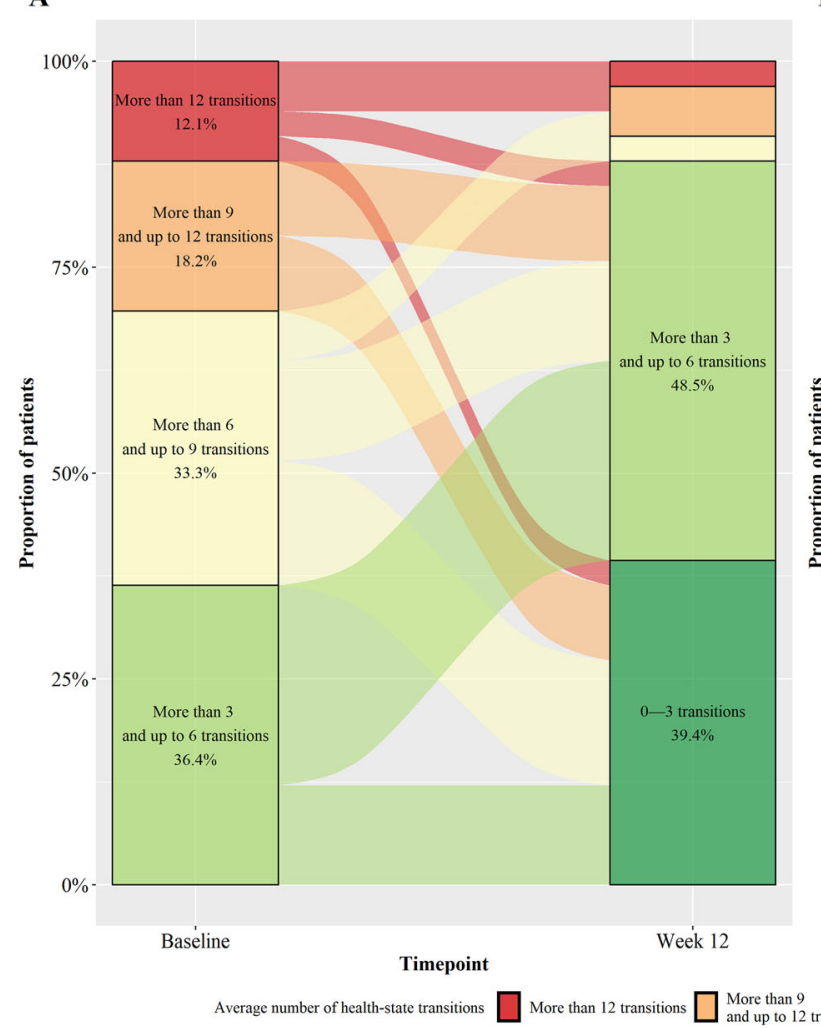

B

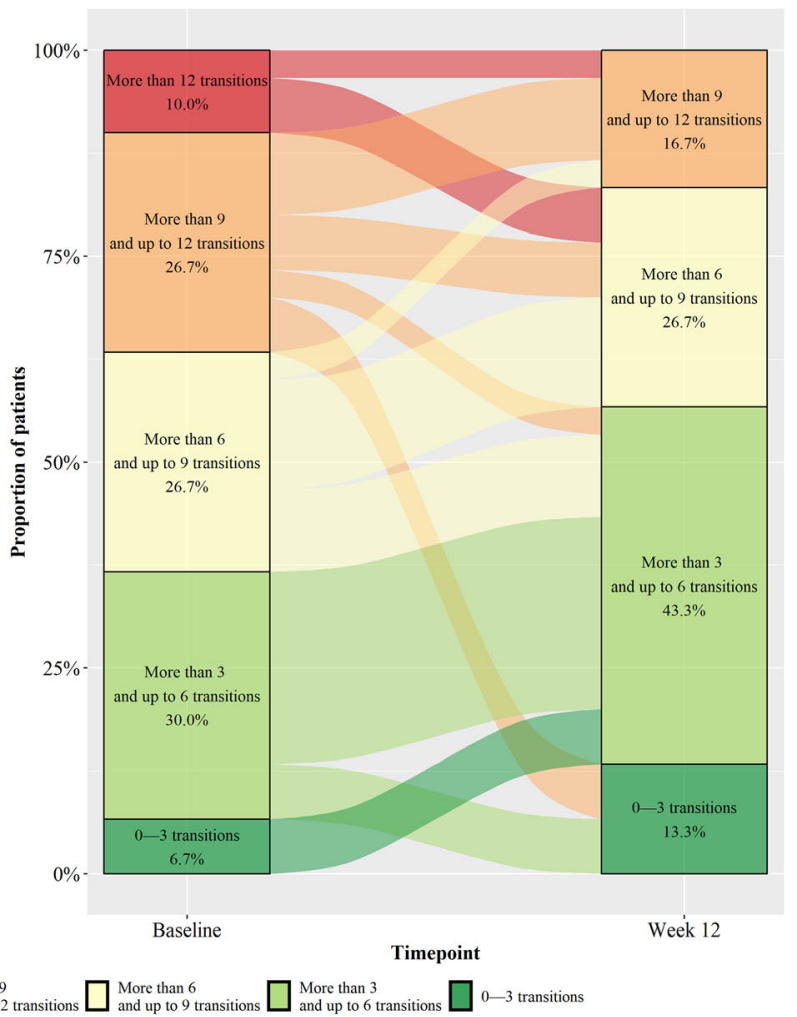

Fig. 4 Average number of transitions between health states during the waking day at baseline vs. week 12. Baseline vs. week 12 for a CLES and $\mathbf{b}$ IR-CL

\section{DISCUSSION}

This is the first analysis of its kind evaluating temporal patterns of motor-symptom control throughout the day with CLES compared to IR-CL. The diary data enabled granular analysis of motor fluctuations and temporal patterns in motor states throughout the day. The phase 3 clinical trial data provided close patient monitoring, follow-up, and well-measured outcomes. Additionally, the randomized study design of the phase 3 trial mitigated bias from potential unobservable differences between the groups. This analysis revealed three main findings in CLES-treated patients compared to patients treated with IR-CL.

First, CLES provides greater and more consistent improvements in OFF and ON-woTD throughout the day than IR-CL. Unpredictability and inconsistency in motor- symptom control constitute well-known limitations of oral therapies that burden patients and their families by limiting patients' ability to plan activities in advance and be on time, decreasing their independence, and inducing changes in social life [19]. By improving consistency in motor-symptom control, CLES treatment may improve both patients' and caregivers' QoL.

Second, CLES was associated with more rapid onset of ON-woTD in the morning than IR-CL. Prior studies have repeatedly found CL oral therapies to provide inadequate benefit for morning akinesia $[8,19,20]$. The advantage of CLES over IR-CL in speed of ON-woTD onset is consistent with CLES's direct infusion into the jejunum $[3,14-16]$. A prior study found that patients receiving subcutaneous apomorphine injection had faster time to ON following morning apomorphine injection than when 
receiving oral levodopa therapy [21]. The results of our analysis are similar in demonstrating benefits of alternative treatment administration methods over oral levodopa treatment in speed of ON time onset. By accelerating ON-woTD onset, CLES may improve patients' QoL, as morning akinesia is correlated with non-motor symptoms and has been found to decrease QoL on several dimensions and to impair ADL, such as the ability to arise from bed, dress, bath, toilet, prepare breakfast, and continue with the day's activities $[8,9,12]$.

Third, CLES-treated patients experienced fewer extreme fluctuations from OFF to ONwTD and had fewer motor-state transitions than IR-CL. In the absence of differences in CL dosing at week 12 between the two groups (demonstrated by the sensitivity analyses), the better patient outcomes with CLES versus IR-CL may be attributed to the difference in delivery modes between the treatments, in that CLES bypasses gastric dysmotility. This analysis thus provides empirical support for the benefit of continuous dopaminergic delivery in terms of motor fluctuations throughout the day $[13,22]$. These benefits are also clinically important given the negative effects of extreme fluctuations and motor-state transitions on patients' well-being [23, 24]. Fewer extreme fluctuations and other motor-state transitions can provide patients with more stability and predictability of their ON state, thus allowing them to more reliably plan ADL and other activities, and hopefully positively impacting their QoL $[9,25]$.

Prior pharmacokinetic studies in the pivotal clinical trials from which this analysis uses data found that as a result of continuous infusion and delivering medication directly into the jejunum, CLES results in lower variability and fluctuations in levodopa and carbidopa plasma concentrations [26]. This provides a possible explanation for the observed results of CLES being associated with more consistent symptom control and more predictable benefit throughout the day than IR-CL.

This analysis is subject to some limitations. First, the data used in this analysis came from a clinical trial consisting of patients that met specific inclusion and exclusion criteria.
Therefore, generalizability of results may be limited, as patients enrolled in the trial may differ from patients with APD in the real world. This concern may be mitigated by the fact that subjects in the trial were very typical of those with APD. Second, IR-CL treatment was optimized to the full extent for patients in the trial, which may not occur in real-world settings; thus, this analysis may overestimate the benefits of IR-CL in real-world settings and underestimate the superiority of CLES over IR-CL. Third, the diary data are patient-reported. As a result, some variation may exist across individual patients' reported motor states because of the patient-reported nature of these outcomes. Finally, as typical with randomized controlled trials involving device-aided therapies, study sample size was somewhat limited, which may have reduced the power for certain analyses. Nevertheless, treatment effects for CLES were still observed.

\section{CONCLUSIONS}

This post hoc analysis provides insight for the first time into the effect of CLES compared to IR-CL on the temporal patterns of patients' motor-states throughout the day. Findings suggest that among patients with APD, CLES provides more consistent symptom control and more predictable benefit throughout the day compared to IR-CL. This can have a profound impact on improving patients' QoL and independence to perform ADLs. Future research should evaluate the effectiveness of CLES on temporal patterns over longer time periods and among a real-world population.

\section{ACKNOWLEDGEMENTS}

Funding. Financial support for the study was provided by AbbVie Inc., North Chicago, IL, USA. AbbVie participated in the interpretation of the data, review, and approval of the manuscript. All authors contributed to the development of the manuscript and maintained control 
over the final content. The sponsor also supported the journal's Rapid Service Fee.

\section{Medical Writing and Other Assis-} tance. Maryaline Catillon, an employee of Analysis Group, Inc., provided assistance with medical writing. Michaela Stamm, an employee of Analysis Group, Inc., provided assistance with the data handling and analysis. Support for these activities was provided by AbbVie.

Authorship. All named authors meet the International Committee of Medical Journal Editors (ICMJE) criteria for authorship for this article, take responsibility for the integrity of the work as a whole, and have given their approval for this version to be published.

Author Contributions. RP, JA, NG, ET, VGH, DS, PLK, VSC, YJJ, YB, PK, and SHI contributed to the study conception and design. Material preparation and analysis were performed by ET, VG-H, and DS. RP, JA, NG, ET, VG-H, DS, PLK, VSC, YJJ, YB, PK, and SHI commented on all drafts of this manuscript. RP, JA, NG, ET, VG-H, DS, PLK, VSC, YJJ, YB, PK, and SHI have read and approved the final manuscript.

Prior Presentation. Portions of this research were presented at the Movement Disorder Society 23rd International Congress of Parkinson's Disease and Movement Disorders on September 12-16, 2020 (Virtual).

Disclosures. Rajesh Pahwa has received consulting fees from AbbVie, Acadia, Acorda, Adamas, Cynapses, Global Kinetics, Lundbeck, Neurocrine, Pfizer, Sage, Sunovion, Teva Neuroscience and US World Meds. He has received research grants from AbbVie, Adamas, Avid, Biotie, Boston Scientific, Civitas, Cynapses, Kyowa, National Parkinson Foundation, NIH/ NINDS, and Parkinson Study Group. Jason Aldred has been a consultant and received honorarium from AbbVie, Acorda, Adamas, Allergan, Boston Scientific, Teva, Medtronic, and US World Meds. He has also received research support from NINDS, Abbott, AbbVie, Acadia, Biogen, Boston Scientific, Denali,
Impax/Amneal, Sunovion, Neuroderm, Novartis, and Theravance. Niodita R. Gupta is a former employee of AbbVie Inc., currently employed by Johnson and Johnson, and may hold AbbVie stock. Vivek S. Chaudhari is a former employee of AbbVie Inc., currently employed by EMD Serono, Inc. and may hold AbbVie stock. Emi Terasawa, Viviana GarciaHorton, and David Steffen are employees of Analysis Group, Inc., which has received consultancy fees from AbbVie. Prasanna L. Kandukuri, Yash J. Jalundhwala, Yanjun Bao, and Pavnit Kukreja are employees of AbbVie and may own stocks/shares in the company. Stuart $\mathrm{H}$. Isaacson has received honoraria for CME, consultant, research grants, and/or promotional speaker on behalf of: AbbVie, Acadia, Acorda, Adamas, Addex, Allergan, Amarantus, Axovant, Benevolent, Biogen, Britannia, Cerecor, Eli Lilly, Enterin, GE Healthcare, Global Kinetics, Impax, Intec Pharma, Ipsen, Jazz, Kyowa, Lundbeck, Michael J. Fox Foundation, Neurocrine, Neuroderm, Parkinson's Study Group, Pharma2B, Roche, Sanofi, Sunovion, Teva, Theravance, UCB, US World Meds, and Zambon. This study was supported by AbbVie, Inc. AbbVie participated in study design, research, data collection, analysis, and interpretation of data, writing, reviewing, and approving the publication.

Compliance with Ethics Guidelines. This article is based on previously conducted clinical trial and does not contain any new studies with human participants or animals performed by any of the authors. The clinical trials conformed with the Helsinki Declaration of 1964, as revised in 2013, and were approved by an independent ethics committee or institutional review board at each participating study site. All patients provided informed consent prior to study participation.

Data Availability. The datasets analyzed during the current study are not publicly available due to patient privacy considerations.

Open Access. This article is licensed under a Creative Commons Attribution-NonCommercial 4.0 International License, which permits any non-commercial use, sharing, adaptation, 
distribution and reproduction in any medium or format, as long as you give appropriate credit to the original author(s) and the source, provide a link to the Creative Commons licence, and indicate if changes were made. The images or other third party material in this article are included in the article's Creative Commons licence, unless indicated otherwise in a credit line to the material. If material is not included in the article's Creative Commons licence and your intended use is not permitted by statutory regulation or exceeds the permitted use, you will need to obtain permission directly from the copyright holder. To view a copy of this licence, visit http://creativecommons.org/licenses/by$\mathrm{nc} / 4.0 /$.

\section{REFERENCES}

1. Politis M, Wu K, Molloy S, Bain PG, Chaudhuri KR, Piccini P. Parkinson's disease symptoms: the patient's perspective. Mov Disord. 2010;25(11): 1646-51.

2. Hauser RA, Friedlander J, Zesiewicz TA, et al. A home diary to assess functional status in patients with Parkinson's disease with motor fluctuations and dyskinesia. Clin Neuropharmacol. 2000;23(2): 75-81.

3. Olanow CW, Obeso JA, Stocchi F. Continuous dopamine-receptor treatment of Parkinson's disease: scientific rationale and clinical implications. Lancet Neurol. 2006;5(8):677-87.

4. Stocchi F. The hypothesis of the genesis of motor complications and continuous dopaminergic stimulation in the treatment of Parkinson's disease. Parkinsonism Relat Disord. 2009;15(Suppl 1):S9-15.

5. Chou KL, Stacy M, Simuni T, et al. The spectrum of "off" in Parkinson's disease: what have we learned over 40 years? Parkinsonism Relat Disord. 2018;51: 9-16.

6. Grosset KA, Bone I, Grosset DG. Suboptimal medication adherence in Parkinson's disease. Mov Disord. $2005 ; 20(11): 1502-7$.

7. Jankovic J. Motor fluctuations and dyskinesias in Parkinson's disease: clinical manifestations. Mov Disord. 2005;20(Suppl 11):S11-6.

8. Rizos A, Martinez-Martin P, Odin P, et al. Characterizing motor and non-motor aspects of early- morning off periods in Parkinson's disease: an international multicenter study. Parkinsonism Relat Disord. 2014;20(11):1231-5.

9. Chapuis S, Ouchchane L, Metz O, Gerbaud L, Durif F. Impact of the motor complications of Parkinson's disease on the quality of life. Mov Disord. $2005 ; 20(2): 224-30$.

10. Damiano AM, McGrath MM, Willian MK, et al. Evaluation of a measurement strategy for Parkinson's disease: assessing patient health-related quality of life. Qual Life Res. 2000;9(1):87-100.

11. Khlebtovsky A, Rigbi A, Melamed E, et al. Patient and caregiver perceptions of the social impact of advanced Parkinson's disease and dyskinesias. J Neural Transm (Vienna). 2012;119(11):1367-71.

12. Tambasco N, Simoni S, Nigro P, Paolini Paoletti F, Marsili E, Calabresi P. Morning akinesia in Parkinson's disease: challenges and solutions. Res Rev Parkinsonism. 2016;6:57-63.

13. Antonini A, Odin P. Pros and cons of apomorphine and L-dopa continuous infusion in advanced Parkinson's disease. Parkinsonism Relat Disord. 2009;15(Suppl 4):S97-100.

14. Nyholm D, Askmark H, Gomes-Trolin C, et al. Optimizing levodopa pharmacokinetics: intestinal infusion versus oral sustained-release tablets. Clin Neuropharmacol. 2003;26(3):156-63.

15. Pfeiffer RF, Isaacson SH, Pahwa R. Clinical implications of gastric complications on levodopa treatment in Parkinson's disease. Parkinsonism Relat Disord. 2020;76:63-71.

16. van Wamelen DJ, Grigoriou S, Chaudhuri KR, Odin P. Continuous drug delivery aiming continuous dopaminergic stimulation in Parkinson's disease. J Parkinsons Dis. 2018;8(s1):S65-72.

17. Olanow CW, Kieburtz K, Odin P, et al. Continuous intrajejunal infusion of levodopa-carbidopa intestinal gel for patients with advanced Parkinson's disease: a randomised, controlled, doubleblind, double-dummy study. Lancet Neurol. 2014;13(2):141-9.

18. Hauser RA, Kremens DE, Elmer LW, et al. Prevalence of dyskinesia and OFF by 30-minute intervals through the day and assessment of daily episodes of dyskinesia and OFF: novel analyses of diary data from Gocovri pivotal trials. J Parkinsons Dis. 2019;9(3):591-600.

19. Chana P, Kuntsmann C, Reyes-Parada M, Saez-Briones P. Delayed early morning turn "ON" in response to a single dose of levodopa in advanced Parkinson's disease: pharmacokinetics should be 
considered. J Neurol Neurosurg Psychiatry. 2004;75(12):1782-3.

20. Stocchi F, Stirpe P. The relevance of dopaminergic level in nocturnal disability in Parkinson's disease: implications of continuous dopaminergic stimulation at night to treat the symptoms. J Neural Transm (Vienna). 2014;121(Suppl 1):S79-83.

21. Isaacson S, Lew M, Ondo W, Hubble J, Clinch T, Pagan F. Apomorphine subcutaneous injection for the management of morning akinesia in Parkinson's disease. Mov Disord Clin Pract. 2017;4(1): 78-83.

22. Nyholm D. Enteral levodopa/carbidopa gel infusion for the treatment of motor fluctuations and dyskinesias in advanced Parkinson's disease. Expert Rev Neurother. 2006;6(10):1403-11.
23. Thanvi B, Lo N, Robinson T. Levodopa-induced dyskinesia in Parkinson's disease: clinical features, pathogenesis, prevention and treatment. Postgrad Med J. 2007;83(980):384-8.

24. Pechevis M, Clarke CE, Vieregge P, et al. Effects of dyskinesias in Parkinson's disease on quality of life and health-related costs: a prospective European study. Eur J Neurol. 2005;12(12):956-63.

25. Pahwa R, Isaacson SH. Pathophysiology, patient burden, and recognition of OFF episodes of parkinson disease. J Clin Psychiatry. 2020;81(6): SU19004BR1C.

26. Othman AA, Rosebraugh M, Chatamra K, Locke C, Dutta S. Levodopa-carbidopa intestinal gel pharmacokinetics: lower variability than oral levodopa-carbidopa. J Parkinsons Dis. 2017;7(2): 275-8. 